\title{
Empowering Responsible Online Gambling by Real-time Persuasive Information Systems
}

\author{
George Drosatos*, Fotis Nalbadis*, Emily Arden-Close*, Victoria Baines*, Elvira Bolat*, \\ Laura Vuillier*, Theodoros Kostoulas*, Sonia Wasowska ${ }^{\dagger}$, Maris Bonello ${ }^{\ddagger}$, Jane Palles ${ }^{\S}$, \\ John McAlaney*, Keith Phalp* and Raian Ali* \\ *Bournemouth University, Talbot Campus, Fern Barrow, BH12 5BB Poole, UK \\ $\{$ gdrosatos,fnalmpantis,eardenclose, vbaines,ebolat,lrenshawvuillier,tkostoulas,jmcalaney,kphalp,rali\}@bournemouth.ac.uk \\ ${ }^{\dagger}$ Sky Betting \& Gaming, 2 Wellington Place, Leeds, West Yorkshire LS1 4AP, UK \\ sonia.wasowska@skybettingandgaming.com

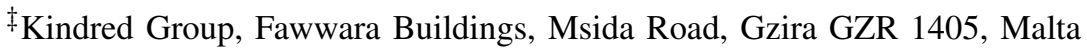 \\ Maris.Bonello@kindredgroup.com \\ §Paddy Power Betfair, Hammersmith Embankment, Chancellors Road, London W6 9HP, UK \\ Jane.Palles@paddypowerbetfair.com
}

\begin{abstract}
Online gambling, unlike other mediums of problematic and addictive behaviours, such as tobacco and alcohol, offers unprecedented opportunities for building information systems that are able to monitor and understand a user's behaviour in real-time and adapt persuasive messages and interactions that would fit their personal profile and usage context. Online gambling industry usually provides Application Programming Interfaces (APIs) meant mainly to enable third-party applications to network with their gambling services and enhance a user's gambling experience. In this industrial practice and experience paper, we advocate that such API's can also be used to retrieve gamblers' online data, such as browsing and betting history, promotions and available offers and use it to build more intelligent and proactive responsible gambling information systems. We report on our industrial experience in this field and make the argument that data available for persuasive marketing and usability should, under specific usage conditions, also be made available for responsible gambling information systems. This principle would provide equal opportunities for both directions. We discuss the psychological foundations of our proposed solution and the risks and challenges typically found when building such a software-assisted intervention, persuasion and emotion regulation technology. We also shed light on its potential implications from the perspectives of social corporate responsibility and data protection. We finally propose a conceptual architecture to demonstrate our vision and explain how it can be implemented. In the wider context, the paper is meant to provide insights on building behavioural awareness and regulation information systems in relation to problematic digital media usage.
\end{abstract}

Index Terms-Persuasive Information Systems, Responsible Gambling, Corporate Social Responsibility

\section{INTRODUCTION}

Online gambling is on the rise and gambling disorder is recognized by the DSM- $5^{1}$. It is easy to access and is empowered by creative information systems able to predict

\footnotetext{
${ }^{1}$ The Diagnostic and Statistical Manual of Mental Disorders, Fifth Edition $(D S M-5)$ is a taxonomic and diagnostic tool published by the American Psychiatric Association (APA) that defines and classifies mental disorders in order to improve diagnoses, treatment and research.
}

the behaviour and personalize the medium and make it more engaging and immersive for users. The ubiquitous accessibility, through desktop and portable devices, makes the scale and complexity of the problem even higher in comparison to traditional gambling which requires going to betting shops. Such mechanisms, along with peer pressure, may prolong exposure, stimulate relapse and hinder attempts to adjust gambling to an acceptable level. Furthermore, the utilization of persuasive techniques (e.g., leader boards and badges) in online gambling forums may increase the risk of gambling to be used as compensation for failure in the real world, mitigate the low-esteem and constitute a replacement for lack of social skills.

However, the characteristics that make online gambling more immersive and engaging, simultaneously also have a great potential to combat problem gambling. The accessibility and persuasive techniques used in online gambling could equally be used when building persuasive information systems to mitigate against the problematic behaviour. Indeed, the cyberspace provides a unique chance to empower classical behaviour change as it offers interactivity, real-time response, traceability of usage data and the support for personalization and context-awareness. Building on the established research in influence [1], help-seeking and behaviour change [2], online addiction labels [3] and persuasive elements of online peer groups [4], we advocate persuasive information systems for assisting responsible online gambling behaviour rather than relying solely on coercive ones. This argument shall also recognize the risk factors when applying such technology for addictive behaviours [5].

Self-regulation information systems are advocated to combat problematic online behaviour given the nature of the medium and the availability of various workarounds when classic and coercive approaches are enacted. Self-regulation systems are centred on the users having an active role in 
changing their own behaviour. An underlying assumption and precondition would be that people would see the benefits of achieving the behavioural change and would, therefore, actively seek help. In addition, such systems when used for regulating online problematic behaviour can take advantages from the medium itself, in order to monitor the behaviour and enact mitigation technologies when problems are happening or about to happen, e.g., interactive warning labels and persuasive interventions such as timers and avatars [3]. An intervention could be designed based on the Theory of Planned Behaviour [6], which holds that attitude to a behavior, subjective norm (perception of how others feel about the behavior) and perceived control over the behavior influence intention to perform the behavior, which ultimately influences whether the individual performs the behavior. Feedback about regularity of gambling and amounts gambled relative to others could help individuals to regulate their behavior, in line with theory around social norms. Similarly, awareness of how behaviour varies across contexts (e.g., making people aware of their gambling varies based on time and place) could increase perceived control over gambling.

There are arguments about the power and risks associated with technology-mediated self-regulation. We still lack strong scientific proof of their effectiveness and also, particularly, of the sustainability of the change they may bring [7]. For example, delivering interventions within peer group settings could potentially be harmful due to factors in relation to group structure and dynamics. This may lead sometimes to reinforcing the negative behaviour [8] and encouraging social loafing [9] or facilitate a temporary change encouraged by the need or pressure to conform [10]. Persuasive technologies may cause individuals to feel frustration, anxiety, intense peer pressure and guilt when not performing or having to cheat the system [11]. This suggests that despite the tempting nature of using online gambling behaviour data in order to build persuasive information systems meant to aid gamblers to regulate their gambling, there is a risk that the change may go in unpredictable directions or be costly in terms of triggering other behaviours.

In this industrial practice and experience paper, we argue the case for supporting responsible online gambling with persuasive information systems that make use of the gamblers' online behavioural, emotional and profile data. Our argument is based on the practical experience we have in the context of the EROGamb project ${ }^{2}$ supported by three main gambling operators in Europe and two problem gambling recovery centres in the UK. The project is funded by a major independent charity for commissioning gambling research in the UK.

The paper is structured as follows. We discuss psychological foundations in behaviour change and their manifestations when applied to online and real-time style in Section II. We give a particular focus to the role of behavioural data which can infer the emotions and mental state of the gambler and how this can

\footnotetext{
${ }^{2}$ EROGamb Project: https://research.bournemouth.ac.uk/project/erogamb/
}

be used for persuasive information systems in Section III. We discuss concerns in relation to commercially sensitive data and corporate social responsibilities in Section IV. We then discuss the rights of users to retrieve their behavioural data and enable their persuasive responsible gambling software in Section V. We present a conceptual architecture of such information systems in Section VI. Finally, we discuss the current stand of the gambling industry in this area and the challenges and risks associated with it in Section VII, and present reflections and future research directions in Section VIII.

\section{REAL-Time PERsuasive Intervention: PSYCHOLOGICAL GROUNDS}

Digital technologies and their information systems allow for new avenues towards behaviour change and prevention strategies. It has been demonstrated for example that digital monitoring of behaviour and automated real-time intervention can successfully be used to encourage drivers to stay within speed limits [12]. This quantification of behaviour allows for the enhancement of several established behaviour change strategies.

The success of interventions and extrinsic motivation, facilitated by techniques such as gamification, may require preliminary processes. First education may be necessary in order to strengthen intrinsic motivation [13] for the behaviour in question (desire to carry out a behaviour based on internal rewards). Intrinsic motivation can be strengthened by requiring individuals to select their individual reasons (from a list) for performing a particular behaviour. Given the interactive nature of the online medium, users could request to be reminded of those reasons on screen at key points while engaging in a particular behaviour, rather than having to write them down.

Goal setting theory is used within behaviour change to encourage individuals to strive to achieve a certain outcome, which may itself involve a series of behavioural changes. This technique is most successful when the behavioural changes are measurable or observable [14], which is particularly relevant to behaviours that can be tracked and shared through the use of digital technologies. Goal setting can be guided by rules such as the SMART criteria. This holds that goals are most likely to be met when they are specific (target a specific area for improvement), measurable (quantify indicators of progress), achievable (can realistically be met by the setter), relevant (apply to the situation in question) and time-bound (are required to be completed within a certain amount of time) [15]. Combined with this, advice on developing implementation intentions (in situation Y, I will do goal-directed behaviour $\mathrm{X}$ ), have a strong significant effect on goal attainment and are effective in promoting goal striving, preventing goal pursuit from unwanted influences and reduction in disengagement [16].

An important part of goal setting is to provide feedback to the individual on how well they are progressing towards their goal [15]. If it is correctly done (e.g., individuals are given feedback that is tailored to their progress, constructive and focuses on behaviours and strategies) this can increase 
the individuals self-regulation or ability to control their own behaviour and sense of self-efficacy [15], which refers to how much they believe that they are capable of changing their own behaviour, and further motivate them to achieve behaviour change. In offline settings however, the opportunities for the individual to receive feedback on their process may be limited; for instance, someone aiming to reduce gambling activity may only receive feedback during weekly meetings with a counsellor or support group. In contrast, feedback on progress that is being measured continuously can be delivered immediately. Indeed, the feedback may even be delivered whilst the behaviour in question is occurring, if the technology is able to detect this. This ability to intervene in real-time with the behaviour is a novel one in the field of behaviour change, where interventions and feedback to individuals is typically asynchronous and occurs after the behaviour has occurred.

Goal setting techniques can be further enhanced through appropriate use of social comparisons as a persuasive tool, which digital technologies are again particularly suited to support. Leader boards can be used to present individuals with information about how their status and progress compare to others within a group. Digital technologies can be used to not only provide individuals with continual and easy access to the leader boards but can also be used to tailor the group membership to that which is the most appropriate for them. It has been demonstrated that individuals will tend to set a goal of being near the top of a leader board if they are presented with one [17]. Signifiers of success and social status can also be used to enhance behaviour change, such as through the use of digital badges to indicate that an individual has achieved a particular goal [17]. This relates to social norms, which refers to what people perceive as typical behaviours, attitudes and aspirations of those within their peer groups. However, these norms can be significantly misperceived and overestimated within groups, particularly those involved in risky or addictive behaviours [18]. Challenging and correcting these misperceptions through social comparisons has been used to bring behaviour change techniques to problematic gambling [19].

Technology can increase the potential of behaviour change techniques, but also the potential for unintended consequences as well. We suggest some potential ways to prevent this below with the study of emotions involved in gambling and persuasive information systems.

\section{Emotions Regulation \& PeRsuading RESPONSIBLE GAMBLING}

In Section II, we discussed how persuasive information systems can be involved in changing the behaviour of gamblers. However, in order to successfully change behaviour, one needs to understand the emotions involved while gambling online. Emotions are intrinsically involved in gambling, from the motivation to gamble to the emotions involved in winning and losing. For example, gambling is thought to increase arousal, decrease negative emotions, or involved in gaining a sense of achievement [20], [21]. Moreover, the emotions relevant to the success of persuasive technology to change gambling behaviour (e.g., the frustration of losing an promotion offer or guessing a result in an in-play betting) need to be assessed in order to make sure this technology is used to facilitate and not impair - behaviour change. Despite their fundamental role, research on emotions in the gambling literature is scarce. Particularly, it is currently unclear: (a) what type of emotions are experienced during different phases of gambling (b) which are the ones particularly associated with the sense of lack of control, and (c) what is the best way to capture them. The latter point is particularly important as individuals with a gambling addiction tend to report high level of alexithymia, manifested by difficulties with identifying and describing one's emotions [22], [23]. Therefore, the development of methods to objectively measure these emotions is necessary [24], [25].

A combination of different multimodal measures, such as facial expressions and galvanic skin response, can help us better understand the role of emotions in gambling. From an affective computing point of view, attempting to capture gambling emotions results in several open issues that need to be investigated, such as the synchronous capture of physiological and behavioural signals and the identification of the most important modalities for emotion detection while gambling [26], [27], [28], [29]. Moreover, we need to identify how the different modalities correlate during an experience, e.g., the correlation between galvanic skin response and facial expressions (intrapersonal correlations) [30].

Besides their role in gambling, emotions have their own place in persuasive information systems. For instance, sending emotionally stimulating messages (such as reminding the gambler of familial impact or longer term financial issues) may help facilitate responsible gambling [31], particularly if it is delivered at the right now, in synchrony with their online gambling behaviour. Similarly, sending personal feedback to online gamblers seems to reduce time and money spent on gambling [32] and it could be worth exploring whether sending this feedback at a particular time during the gambling process (such as during loss chasing or denial) may influence the emotions involved and helps further increase responsible gambling. These questions need yet to be investigated. The rise in smartphone technology and its potential use in psychiatry and health behaviour [33] means that this is the perfect time to do so. Indeed, one could hope that smartphone technology will help with the automatic identification of physiological, emotional and behavioural signals, so that persuasive techniques can be implemented at the right time, when the individuals are in the right emotional state, and with the right level of motivation. This could help prevent some of the unintended consequences of persuasive technology.

\section{Corporate Responsibility Vs. Commercially SENSITIVE DATA}

The allowance of gambling operators of the retrieval of online gambling behaviour data by responsible gambling software can prove a higher degree of corporate social responsibility but at the same time raise issues around the commercial 
sensitivity of these data especially when the software is operated by third parties. Technological advancements have empowered online gambling with vast amount of data to tailor immersive gambling experience and to also aid responsible gambling decision making [34], [35]. Online gambling operators are deemed to act responsibly to manage societal expectations and controversial perceptions of the gambling industry and in particular tackle issues of problem gambling [36]. In this process, data management and sharing are pivotal to corporate social responsibility (CSR) and duty of care conduct. However, gambling operators, as commercial entities, may be wary of engaging with a responsible data sharing process, and may consider customer data, for instance, their customers' behaviour, as commercially sensitive. Making commercially sensitive data available in this manner may simply be viewed by gambling operators as risking their competitive advantage. We argue that in the long run data sharing presents an advantage to the in industry, hence, a balanced approach to data sharing is necessary.

Literature argues [37] that any data and information that has economic value or potential to cause economic harm to an organisation can be classified as commercially sensitive data. Commercial organisations often use a term 'trade secret' as a synonym to a commercially sensitive data. A trade secret is defined in [38] as:

"Any formula, pattern, device, or compilation of information which is used in one's business, and which gives the holder an opportunity to obtain an advantage over competitors who do not know or use it."

The underlying logic of commercially sensitive data is that some data requires protection and 'secret' status to forest innovative business practices [38]. Examples of such data in online gambling industry are marketing strategies, consumer lists and online behavioural data. However, we argue that it is data usage, not data sharing, that generates a competitive value.

The counter argument is that since the financial terms of many online gambling products are known within the industry, the argument that data availability and transparency would cause competitive harm seems weak. In fact, a disclosure of anonymised consumer lists or behavioural data may be required to serve a greater societal interest [39]. This is particularly applicable to the online gambling context. Clearly while making profits is the core business need for gambling operators, they are expected to protect gamblers, and detect vulnerable and problematic behaviour [36]. In fact, responsible gambling strategies resulting from credible use and sharing of data can form an individual operators' competitive differentiation as well as change of the industry's image and lead to sustainable business growth.

Corporate social responsibility (CSR) in a digital era is a subject to establishment due to new areas of digital technology applications such as the use of artificial intelligence in customer services and in the profiling of consumers. As laws fail to keep up with speed of technological changes, ethical practices have to be developed to establish moral principles of using data. Conversations around ethical big data are contributing towards a shared understanding of ethical, social, and legal complexities of data analytics [40]. Moreover, within the online gambling discipline there is a movement towards establishing CSR principles and encouraging data sharing and transparency. For instance, academic researchers [41], [42] are exploring ethical recommender systems to provide guidance on the moral norms for serving recommendations of online gambling products to players. Although such ethical recommender systems research is in its infancy stage, it triggers industrywide conversations about online gamblers' moral obligations to prevent mis-selling, behavioural manipulations and privacy intrusion [43].

Despite the above listed regulatory and conceptual efforts in framing CSR principles in a digital era, the complexity of organising and managing CSR practices requires a collaborative approach to data sharing and management, at least within a single operator's context if not across the industry. In particular, we argue the case for a principle that data made available for marketing divisions and their software would need to be equally, subject to usage conditions, available to responsible gambling divisions and their surrogate software. Responsible gambling divisions can then enact certain configurations for each sector of the gamblers. The principle is to provide equal opportunity for both directions and leave the decision to the gamblers themselves. Following this principle, gambling operators are managing all three elements crucial to an excellent customer experience and compliance process: entertainment, accessibility and risk.

\section{Data for Persuasion vs. Data Protection REGULATIONS}

In order to share online gambling behavioural data with responsible gambling persuasive software, gambling operators also need to comply with data protection regulations. The gambler's permission to such software to act on their behalf in retrieving these data for offering the assistance in the behaviour change raises a range of legal concerns. In this section, we discuss this critical issue for the success of such technology from the perspective of a major data protection law, the European Union General Data Protection Regulation (GDPR), which was adopted on 27th April 2016 [44]. Online service providers are occupied with ensuring that they are prepared for GDPR's requirements before the implementation deadline of 25th May 2018. These include the provision of privacy impact assessments, procedures for subject access requests and subject requests for the erasure of personal data (the so-called "Right To Be Forgotten").

Against this backdrop of compliance pressure, it may be tempting for providers to assume that the requirement for data minimisation trumps all others. While at the time of writing some uncertainty remains over exactly how GDPR's provisions will be transposed into the national legislations of EU Member States, the headline provisions for the processing and 
collection of personal data are universal. Particular emphasis is placed in the GDPR on 'lawfulness, fairness and transparency' in relation to data processing (II.5.1(a)).

GDPR does not specifically prohibit further processing or indeed transmission to a third party recipient, provided that information concerning "the purposes of the processing for which the personal data are intended as well as the legal basis for the processing" and "the recipients or categories of recipients of the personal data, if any" is adequately communicated to the subject (13.1(c),(e)). Many online service providers already list these purposes and recipients at least in general terms in their published data use and privacy policies.

In response to subject access requests, providers will be required to show greater transparency also concerning "the existence of automated decision-making, including profiling, referred to in Article 22(1) and (4) and, at least in those cases, meaningful information about the logic involved, as well as the significance and the envisaged consequences of such processing for the data subject" (15.1(h)). As a result, companies may come under greater pressure to reveal commercially sensitive data in order to meet their obligations under data protection law. GDPR likewise gives EU citizens the Right to Object to being profiled, including where this is for direct marketing.

Far from being a merely restrictive instrument, GDPR therefore empowers EU citizens to have more information about and greater input over what happens to their data. The Regulation's emphasis on obtaining explicit consent for data processing and transfer affords the opportunity for online gambling providers to offer an "opt in" model for behavioural analysis with the aim of improving public health. Giving players the choice of whether to have their activities analysed in this way, giving them the power to withdraw that consent, and clearly articulating how such data would be processed and transferred would be in line with the data protection regime as currently set out in GDPR.

The provisions of GDPR apply not only to EU Member States, but also to all service providers that process EU citizens' data. In the global marketplace of online gambling, its obligations therefore extend far beyond the boundaries of the EU. Third countries are naturally looking to align their national legislations with GDPR, not least because of its provisions concerning international data transfers (Articles 44ff.). Sector specific legislation also does not prohibit the sharing of personal data for research and public health purposes. Rather, the Gambling Act 2005 states simply that data disclosures must not contravene the Data Protection Act 1998 (c. 29) [45].

In light of governments' increasing requirement for social responsibility from licensees, designation of the development of more effective harm minimisation interventions as a priority objective of the UK National Responsible Gambling Strategy, and evidence of an appetite among online gamblers for tools that aid spending limit setting, self-diagnosis of problematic behaviours and self-exclusion, analysis of data protection legislation reveals the time is arguably ripe for data sharing by online gambling providers expressly for the development of public health tools [46], [47].

\section{Vi. Proposed Architecture for Persuasive RESPONSIBLE GAMBLING INFORMATION SYSTEMS}

The conceptual architecture of our vision is presented in Figure 1. In order to cover all of the requirements, debates and issues that were outlined in the previous sections, we propose a hybrid approach that constitutes two complete subsystems: (1) an end user intelligent agent and (2) a research platform for responsible online gambling. The second subsystem is responsible for supporting research about persuasive technologies that could be applied to users with addictive behaviour. After a testing and evaluation period the findings (from (2)) could be then feed the end user intelligent agent. The end user intelligent agent can autonomously run to a large extent and is responsible for guiding the gamblers to self-regulate their problematic behaviour. Its main advantage is that it provides high privacy guarantees to the users because the processing and the storage of data happens on the user-side without leakages to any third-party system.

In more detail, the research platform architecture consists of the following:

- Central repository: The central repository is responsible only for storing users' data for research purposes. This data can be separated into two main categories, (a) online gambling experience data and (b) multimodal data. The first category is about data that is recorded by gambling operators, such as, betting history, spending time, amount of money and online status (more details about the availability of this data is discussed in Section VI-B). The second category is about data that is captured by the personal devices of the user, such as geolocation, accelerometer data, heart rate and eye tracking data. The stored data in this repository would be derived from many users and many operators to cover a holistic view of users' gambling activity. All of this knowledge is important for research and development reasons, but simultaneously it is also risky for privacy, commercialisation and unfair competition reasons. For this reason, this data should be stored for small period, under operators and users' agreements, and for small-scale application.

- Client for operator's API: A background service that manages users' credentials (e.g. OAuth 2.0 authorization framework) and retrieves users' gambling activity from online gambling operators' APIs (Application programming interface). The frequency of requests to these APIs is crucial in order to support real-time intervention to gamblers. Ideally, a push API would be the best option for our platform but based on our knowledge such APIs are not provided by gambling operators for public use.

- API for incoming data: This API is provided by our platform to collect multimodal sensors' data from users' personal devices. Thus, an app/service that is running in user's mobile phone collects data from the available sensors (i.e. accelerometer, GPS, camera, microphone, etc.) and sends it to our platform. Apart from this data, we could also collect data from wearable sensors (i.e. 


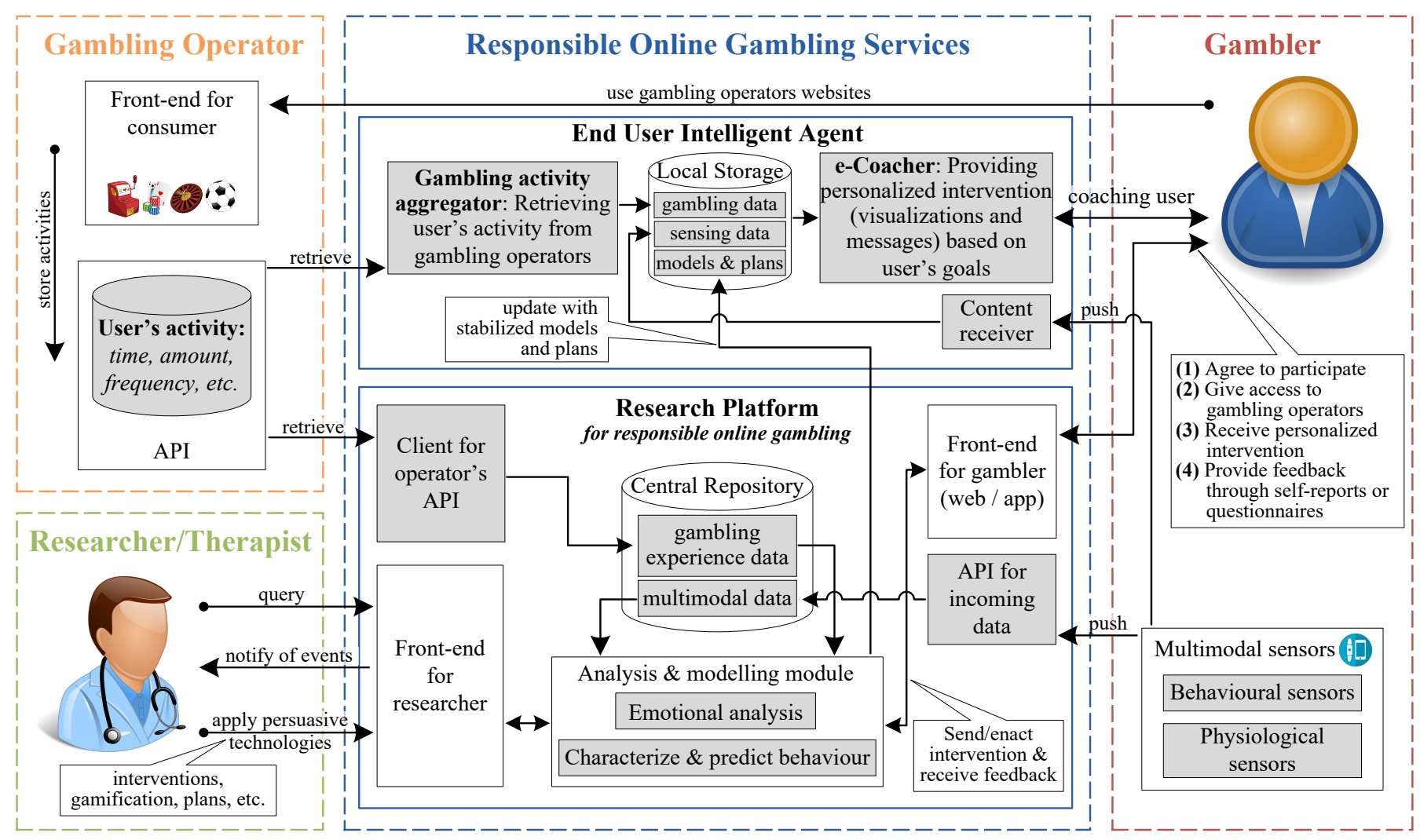

Fig. 1. The conceptual architecture for responsible gambling persuasive information systems

smartwatch, heart rate, blood pressure, etc.) that store their data to manufactures' cloud (e.g. Fitbit).

- Front-end for researcher/therapist: The researchers' front-end constitutes a web-based interface where the researcher or therapist can monitor the user's activity, receive notifications when an abnormality detected on user's behaviour and manage what interventions, gamifications and plans can be applied to each user or group of users. In this interface, each user has a unique identifier that is not linked for privacy reasons with the real identity of user and it is used to trace users' activity.

- Front-end for gambler: The gamblers' front-end could be a web interface and/or a mobile application where the user could watch their betting activity (i.e. time, money, bets, etc.), set the goals that they want to achieve for a healthier gambling activity and receive interventions through appropriate visualizations and messages. All of these functionalities are required to self-regulate their behaviour. In addition to the feedback that can be captured from the gambling activity of the user, our research platform also requires feedback directly from them, in the form of self-reports or even questionnaires. This feedback will be used by the researchers to adjust the applied persuasive technologies and predictive models.

- Analysis and modelling module: This module is the core of our research platform and is responsible for performing all of the required analysis and modelling of users' multimodal data based on the researchers' directions. This module consists mainly of two sub-components:

- Emotional analysis: This subcomponent is responsible for processing and analysing the signals captured from the corresponding sensors and for creating and utilizing the emotion models. Furthermore, it has to deal with time synchronization of all modalities and alignment of the measurements [26], [48], [28]. The challenge in the development of the emotion models relies on accounting for the different reaction times for different modalities, the different duration of different emotions and periods of loss of control, as well as the available data for building the corresponding models.

- Characterize \& predict behaviour: In this subcomponent, the extracted emotional data from the previous subcomponents combined with gambling experience data will be utilized to characterize and predict the behaviour of gamblers. The result of this process would be provided as personalized intervention (i.e. visualizations and notifications) to the users with the goal of informing and empowering them, and ultimately helping them to change their behaviour.

Finally, after a testing and evaluation period the generated models and algorithms will be used in our end user intelligent agent that works autonomously and focuses on the general population of gamblers. 
Accordingly, the end user intelligent agent, based on the current technological developments, could be served as a mobile application or even a client-side web application. The usage of smartphones as a platform to serve our agent through a mobile application could be the best option, because it is a well-known and powerful computing platform, is always together with the user and embeds many sensors. Following the description of components that compose the end user intelligent agent:

- Local storage: The local storage is a small database inside our agent that only stores the personal data of user and knowledge about the last up-to-date predictive models. These models are provided, only when previously validated and confirmed, by our research platform.

- Gambling activity aggregator: A service that runs in the background and aggregates users' gambling activity from online gambling operators' APIs. Additionally, it could occasionally run when a network is available and with a fixed frequency of requests.

- Content receiver: The content receiver constitutes an interface that gives access to sensor data (e.g. geolocation and accelerometer) from the user's personal device and/or retrieves data, through content providers or an API, from other third-party applications that manage wearable sensors (e.g. Fitbit).

- e-Coacher: The e-Coacher is the core component of the end user intelligent agent and is responsible for applying the available predictive models and algorithms on the user's data. The results of this processing are then used to provide personalized intervention through visualizations and notifications. This personalization happens using the user's profile data, gambling activity and the goals that user has set for themselves. Thus, the gambler has a continuous interaction with his e-Coacher to help him achieve a healthier gambling behaviour.

\section{A. Authentication Procedure for Gamblers Participation}

Privacy, informed consent and authentication are major challenges facing the feasibility of our architecture. This is mainly about the sharing of online gambling behavioural data with third parties (e.g. research or therapists, who recruit gamblers to participate in a study or a trial) even when authorized by the gamblers and reviewed by the gambling operator for their intent and declared usage of data. In order to assure the gamblers' participation in the platform, we propose a privacy by design process that assures the gambling operators about the gamblers' intent to participate in the platform and ease the handling of anonymity and the effect of data leak especially when storing the retrieved gambling behavioural data at the third party side or cloud storage. The overall process is presented in Figure 2. A detailed description of this process is presented as follow:

- The moderator (researcher or therapist) using the platform shall start by inviting participants who meet the suitability criteria to participate.
- The gamblers, who want to participate in the platform, shall first sign the consent form and provide their account of gambling operator. They will then be given a random alphanumeric as a unique identifier for the particular study or therapy.

- The moderator, researcher or therapist, will have then to provide the gambling operator (through a dedicated web page or programmatically through an API) with the unique identifier for the account.

- After that, the gamblers shall be instructed to login with their account into the gambling operator and visit a designated web page in the gambling site itself where they have to enter the given unique identifier. If it matches their account, they will be asked if they agree to take part in the platform and share their data (for a specific time frame, including the past) with the moderator. At the end of this process, the gambling operator will notify the research platform about the new participant.

- At any time, the gamblers can visit to the same page and select to stop the monitoring process preventing the moderator from seeing their gambling behaviour. Also, they can select to revoke access to the data already collected and made available to the moderator. In both cases, the platform will be notified by the gambling operator for the change in status.

- The gamblers can then use the site and app offered by our platform by entering their unique identifier. None of the messages sent to them will contain personally identifiable information.

This protocol seems to be acceptable by the various parties involved in the process, i.e. the moderators, the gamblers and the gambling operators. The reliance on the strength of the authentication process at the operators' side is a key part of it. The storage of anonymised data at the platform side where the mapping between the key and the real identities is stored separately is also additional assurance against data leak and its effect.

\section{B. Gambling Operators Data Availability}

The online gambling operators currently provide infrastructures where users can access their personal data. These infrastructures are mainly constituted from publicly available APIs and data exporting mechanisms via the operators' websites (e.g. downloaded as *.csv files). According to the European and the UK data protection regulations (see more in Section V), each user has the right to know what data and under what circumstances an operator keeps data about them and also has the right to access their data. Unfortunately, the existing infrastructures only provide some of the user's personal data that describe their online gambling activity. This data mainly includes the following:

- Betting history: A list of the user's bets that contains data such as: date and time, a general description of the bet, status of the bet (won or lost), stake and pot return.

- Account statements: A list of transactions in the user's virtual wallet that contains data such as: date and time, 


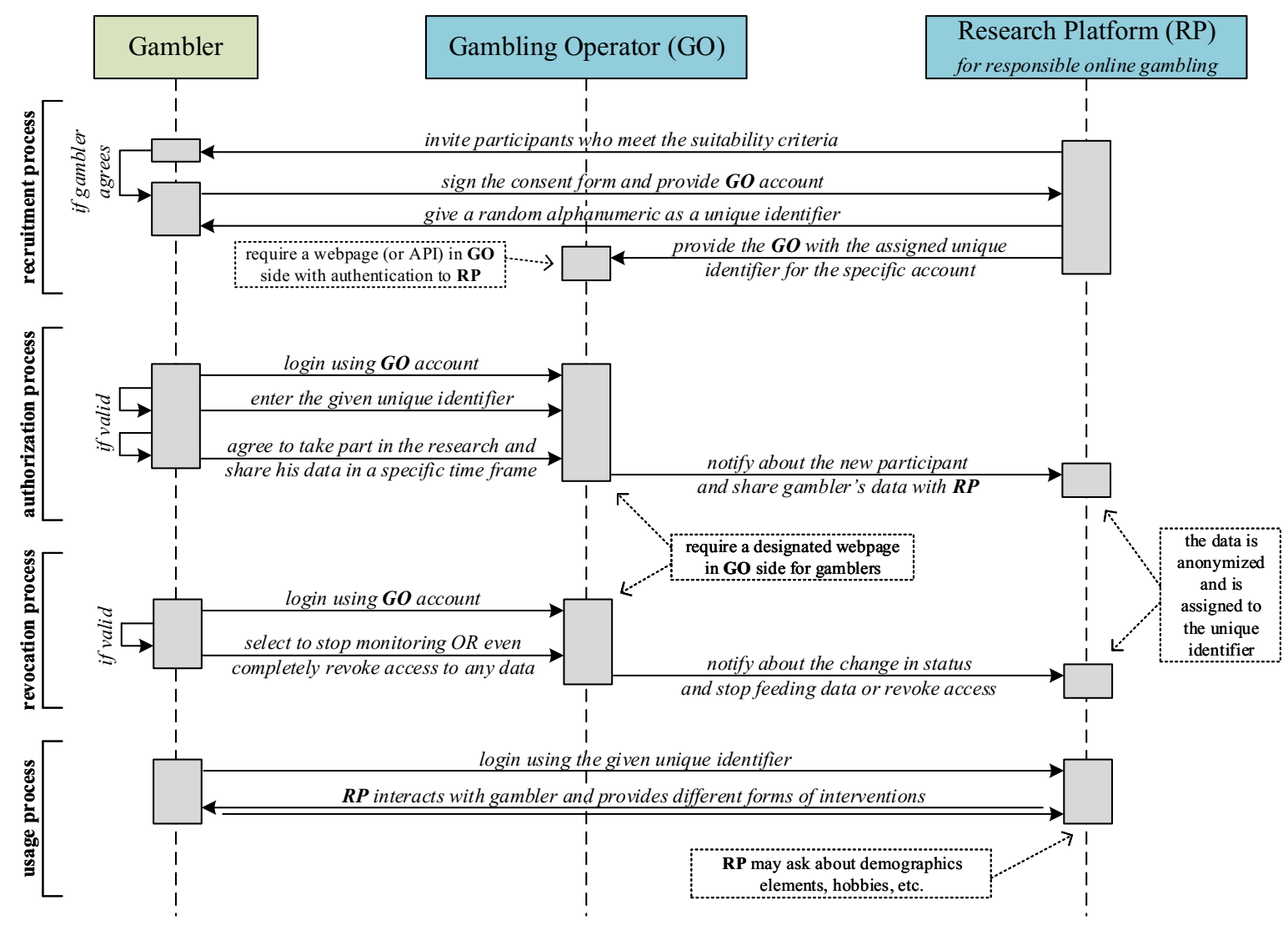

Fig. 2. The sequence diagram of a consensus procedure for gamblers participation

description of the transaction (e.g. casino, sportsbook, etc.), debit or credit amount and balance.

These types of data could be used in our system, in addition to informing the user about their betting activity and spending, to extract knowledge about the user's addiction level in gambling, whether there are any increasing or decreasing trends and time (without good accuracy) consumed in gambling. However, all this of data is not sufficient to enable precise, smart and meaningful intervention to the users. Thus, it is crucial for the gambling operators to enrich their infrastructures (and especially APIs) by giving more details about users' gambling activity. Note that this would be data that they already record for internal reasons (e.g. security and marketing). For example, this additional data could be:

- Additional details about betting history: This includes the activities with the date and time, game type and events type where the user places their bets (i.e. football, Spanish league, top popular, etc.). Additionally, it would be good to know the device used to access the operator (mobile app or website), the user's location, whether a bet was cashed out, the time of the event and its location. This detailed betting history could then be used by our system in order to provide more appropriate messages for the current user's context and to predict, for example, how the user bets in comparison with his location. Thus, this inside information could make the user to change behaviour more easily.

- Real-time navigation behaviour in operators' platforms: This includes information about the state of the user (i.e. logged in or logged out), the sections visited in the operators' platforms (e.g. pages such as bingo, account and history, sportsbook, etc.), the navigation spending time and the links clicked which redirect to third party web pages (e.g. responsible gambling sites, etc.). In addition to the previous required data from operators, the real-time navigation behaviour of the user could help us provide messages or notifications in appropriate times, for example, just before placing a new bet.

\section{Discussion And Research Challenges}

Gambling businesses develop complex strategies to analyse and utilise customer data. Maximising user experience, marketing, trading and overall commercial strategies have been the predominant goals of individual operators. At the moment, considering the ever growing regulatory burden as well as increasing social responsibility awareness, there is more appetite than ever to engage in collaborative projects to research and utilise online data for player protection purposes [49], [47]. Responsible gambling is acknowledged as an area of need and given the variety of data available, it offers a considerable opportunity to link problem gambling identification with appropriate communications, timed and constructed to 
suit individuals and their particular circumstances. There is an acknowledgment of challenges around sharing personal data and obtaining relevant permissions on a legal level, as well as the competitive nature of the industry, however in consideration of benefits to be gained by engaging in this area, not only to facilitate a safer gambling experience for the individual players but also support industry-wide responsible gambling strategies. Maintaining a healthy balance between commercial and responsible gambling imperatives can be achieved by relevant investment in research and technological solutions supportive of both facilitating more informed decision making as well as staged interactions and interventions programmes.

Whilst digital technologies can be used to facilitate and improve behaviour change strategies there are also risks to be considered. One of these risks relates to unsustainable change. Goal setting theory holds that, learning goals relate to improving competence by targeting skills and acquisition of knowledge, whereas performance goals focus on rewards to provide a sense of accomplishment [50]. Learning goals are more effective in bringing about sustained behavioural change, as they can motivate goal pursuit when dealing with failure [51]. In the short term, individuals are more likely to be motivated by performance goals, which may have the unintended effect of decreasing motivation. For example, if an individual is consistently at the bottom of a leader board, they may develop a sense of low self-efficacy, in which they cease to believe they are capable of achieving behaviour change. In keeping with social psychological theories, such as learned helplessness [52], this may mean that the individual stops even attempting to change and disengages with the process. Similarly, attempts to promote a social norm within a group may be counterproductive if there is a sub-group which defines itself by behaving in an extreme manner, as has been found to be case with alcohol use in some populations [53].

Another issue is loss of interest. While online interventions have the potential to reach a wide population, attrition is common and tends to occur rapidly [54]. The challenge is how to enable sustained behaviour change.

Another threat is failure to engage. This may be due to negative feelings individuals experience when they do not receive expected support from peers. Careful expectation management is necessary in order to manage such risks.

Comparison techniques are a part of many digital interventions. Comparisons are made with previous behaviour, peers or in relation to goals set. Comparing behaviour to past behaviour or behaviour of others is meant to enhance self-esteem and self-efficacy. However, such a strategy may negatively impact those constructs. For example, upward social comparisons (with those with less problematic usage) may lead to an individual feeling depressed and unmotivated [5].

Although an online intervention that encourages people to gamble responsibly would usually be viewed positively, it is possible that such an intervention may become a basis for alternative addictive behaviours [5]. For example, email or text messages from the software may trigger further gambling episodes. It is also possible that having a leader board may result in individuals checking websites more, and becoming obsessed with their position. While this would not cause loss of money in the same way as online gambling, it may become equally problematic with regard to loss of time.

While allowing individuals to set their own goals can enhance self-efficacy, SMART goals are difficult to set. In the development of a web-based weight loss intervention, participants struggled with setting SMART goals [55]. Users may be influenced by previous experiences, and previous experience of setting challenging goals that have led to failure may lead to setting of easy goals. Further, when setting goals in order to achieve a particular position on a leader board, individuals may set easy goals, particularly if they are comparing themselves to those with more problematic usage (downward comparison).

\section{CONCLUSIONS}

This paper is meant to shed light on an emerging paradigm of information systems which is aimed to support behavioural awareness and behavioural change through persuasive and motivational software-based techniques. In particular, we study behaviours which can be tracked, to some extent, through automated means, e.g. online usage and sport activities, etc. We take as an exemplary domain the case of online gambling. We argue that responsible online gambling can be supported by persuasive information systems that make use of the gamblers online behavioural, emotional, and profile data. Such technology can increase the potential of behaviour change techniques, but may also have unintended consequences. To avoid these consequences, developing objective ways of measuring emotions and implementing emotions to the persuasive design has the potential to help develop better, targeted and personalised interventions for individuals with a gambling problem. From the gambling operators' point of view, we would require them to exhibit greater social responsibility, with, in particular, a collaborative approach to data sharing and management.

The paper is based on practical experiences and joint effort with main problem gambling recovery charities and responsible gambling experts in the UK. We are making the point that data, which is often already available to the operators and used for their own business purposes, should be equally available for responsible gambling reasons. Data protection legislation reveals that this is the perfect time for online gambling providers to share their data. To this end, we have presented a conceptual architecture that reveals our vision in the area and demonstrates how such approaches could be developed. However, the main goal of this paper is to provide a starting point for a discussion on these issues, which ideally would involve contributions from computing, psychology and corporate social responsibility perspectives. This would encourage, what we believe is much needed, policy change in this area, or, as a minimum engender a shared corporate view which leads to the provision of the kind of data that could be used to allow systems, such as those described in concept, to mitigate against problematic gambling. 
Despite the focus on online gambling in the paper, much of the presented foundations and debates apply equally to persuasive information systems for combatting problematic online behaviour in general. For example, game addiction, obsessive social media usage, compulsive online shopping are other domains where we can demand that online behavioural data are made available to the users and their surrogate agent, humans like a counsellor or a software. Although such sharing tends often to be encouraged for transparency and informed usage reasons, challenges remain in relation to its potential of affecting users experience and also creating negative sideeffects. This requires more theory-informed utilization of data to design the interaction and persuasive and motivational mechanisms over the change life cycle.

\section{ACKNOWLEDGMENTS}

This work was partly supported by the EROGamb Project (Empowering Responsible Online Gambling with Predictive, Real-time, Persuasive and Interactive Intervention). This project is funded jointly by GambleAware and Bournemouth University. We also thank StreetScene Addiction Recovery and The Gambling Therapy for sharing their practical experiences in the field of problem gambling and providing feedback on this work.

\section{REFERENCES}

[1] L. Davidson, M. Chinman, B. Kloos, R. Weingarten, D. Stayner, and J. K. Tebes, "Peer support among individuals with severe mental illness: A review of the evidence," Clinical Psychology: Science and Practice, vol. 6, no. 2, pp. 165-187, 1999.

[2] R. H. Moos and B. S. Moos, "Help-seeking careers: Connections between participation in professional treatment and alcoholics anonymous," Journal of Substance Abuse Treatment, vol. 26, no. 3, pp. 167173, 2004.

[3] R. Ali, N. Jiang, K. Phalp, S. Muir, and J. McAlaney, The Emerging Requirement for Digital Addiction Labels. Cham: Springer International Publishing, 2015, pp. 198-213.

[4] A. Alrobai, J. McAlaney, K. Phalp, and R. Ali, Online Peer Groups as a Persuasive Tool to Combat Digital Addiction. Cham: Springer International Publishing, 2016, pp. 288-300.

[5] _ _ "Exploring the risk factors of interactive e-health interventions for digital addiction," International Journal of Sociotechnology and Knowledge Development, vol. 8, no. 2, pp. 1-15, 2016.

[6] I. Ajzen, "The theory of planned behavior," Organizational behavior and human decision processes, vol. 50, no. 2, pp. 179-211, 1991.

[7] S. Leigh and S. Flatt, "App-based psychological interventions: friend or foe?" Evidence-Based Mental Health, 2015.

[8] T. J. Dishion, J. McCord, and F. Poulin, "When interventions harm: Peer groups and problem behavior." American psychologist, vol. 54, no. 9, pp. $755-764,1999$.

[9] S. Karau and W. , "Social loafing: A meta-analytic review and theoretical integration," vol. 65, pp. 681-706, 101993.

[10] V. L. Allen, "Situational factors in conformity," Advances in experimental social psychology, vol. 2, pp. 133-175, 1965.

[11] J. Hamari, J. Koivisto, and T. Pakkanen, Do Persuasive Technologies Persuade? - A Review of Empirical Studies. Cham: Springer International Publishing, 2014, pp. 118-136.

[12] I. J. Reagan, J. P. Bliss, R. V. Houten, and B. W. Hilton, "The effects of external motivation and real-time automated feedback on speeding behavior in a naturalistic setting," Human Factors, vol. 55, no. 1, pp. 218-230, 2013.

[13] R. M. Ryan and E. L. Deci, "Self-determination theory and the facilitation of intrinsic motivation, social development, and well-being," American psychologist, vol. 55, no. 1, p. 68, 2000.

[14] G. H. Seijts, G. P. Latham, K. Tasa, and B. W. Latham, "Goal setting and goal orientation: An integration of two different yet related literatures," Academy of management journal, vol. 47, no. 2, pp. 227-239, 2004.
[15] L. K. B. Eldredge, G. S. Parcel, G. Kok, N. H. Gottlieb, and M. E. Fernández, Planning health promotion programs: an intervention mapping approach, 3rd ed. San Francisco, CA: Jossey-Bass, 2011.

[16] P. M. Gollwitzer and P. Sheeran, "Implementation intentions and goal achievement: A meta-analysis of effects and processes," Advances in experimental social psychology, vol. 38, pp. 69-119, 2006.

[17] R. N. Landers, K. N. Bauer, and R. C. Callan, "Gamification of task performance with leaderboards: A goal setting experiment," Computers in Human Behavior, vol. 71, no. Supplement C, pp. 508-515, 2017.

[18] J. McAlaney, B. Bewick, and C. Hughes, "The international development of the 'social norms' approach to drug education and prevention," Drugs: Education, Prevention and Policy, vol. 18, no. 2, pp. 81-89, 2011.

[19] L. Marchica and J. L. Derevensky, "Examining personalized feedback interventions for gambling disorders: A systematic review," Journal of behavioral addictions, vol. 5, no. 1, pp. 1-10, 2016.

[20] T. Ricketts* and A. Macaskill, "Gambling as emotion management: Developing a grounded theory of problem gambling," Addiction Research \& Theory, vol. 11, no. 6, pp. 383-400, 2003.

[21] A. Blaszczynski and L. Nower, "A pathways model of problem and pathological gambling," Addiction, vol. 97, no. 5, pp. 487-499, 2002.

[22] A. D. Williams, J. R. Grisham, A. Erskine, and E. Cassedy, "Deficits in emotion regulation associated with pathological gambling," British Journal of Clinical Psychology, vol. 51, no. 2, pp. 223-238, 2012.

[23] M. A. Lumley and K. J. Roby, "Alexithymia and pathological gambling," Psychotherapy and Psychosomatics, vol. 63, no. 3-4, pp. 201-206, 1995.

[24] L. Lole, C. J. Gonsalvez, A. Blaszczynski, and A. R. Clarke, "Electrodermal activity reliably captures physiological differences between wins and losses during gambling on electronic machines," Psychophysiology, vol. 49, no. 2, pp. 154-163, 2012.

[25] A. Parke, A. Harris, J. Parke, P. Goddard et al., "The role of stake size in loss of control in within-session gambling impact of stake size on reflection impulsivity, response inhibition and arousal when gambling on a simulated virtual roulette gambling task: implications for gambling related harm," 2014.

[26] T. Kostoulas, G. Chanel, M. Muszynski, P. Lombardo, and T. Pun, "Films, affective computing and aesthetic experience: Identifying emotional and aesthetic highlights from multimodal signals in a social setting," Frontiers in ICT, vol. 4, p. 11, 2017.

[27] L. Tian, M. Muszynski, C. Lai, J. Moore, T. Kostoulas, P. Lombardo, T. Pun, and G. Chanel, "Recognizing induced emotions of movie audiences: Are induced and perceived emotions the same?" in 7th International Conference on Affective Computing and Intelligent Interaction (ACII). IEEE, 2017, pp. 1-8.

[28] T. Kostoulas, I. Mporas, O. Kocsis, T. Ganchev, N. Katsaounos, J. J. Santamaria, S. Jimenez-Murcia, F. Fernandez-Aranda, and N. Fakotakis, "Affective speech interface in serious games for supporting therapy of mental disorders," Expert Systems with Applications, vol. 39, no. 12, pp. $11072-11079,2012$

[29] T. Kostoulas, G. Chanel, M. Muszynski, P. Lombardo, and T. Pun, "Identifying aesthetic highlights in movies from clustering of physiological and behavioral signals," in Quality of Multimedia Experience (QoMEX), 2015 Seventh International Workshop on. IEEE, 2015, pp. 1-6.

[30] E. Kroupi, J.-M. Vesin, and T. Ebrahimi, "Phase-amplitude coupling between eeg and eda while experiencing multimedia content," in $A f$ fective Computing and Intelligent Interaction (ACII), 2013 Humaine Association Conference on. IEEE, 2013, pp. 865-870.

[31] A. Harris, A. Parke, and M. D. Griffiths, "The case for using personally relevant and emotionally stimulating gambling messages as a gambling harm-minimisation strategy," International Journal of Mental Health and Addiction, 2016.

[32] M. M. Auer and M. D. Griffiths, "The use of personalized behavioral feedback for online gamblers: an empirical study," Frontiers in psychology, vol. 6, p. 1406, 2015.

[33] J. Torous, "Digital psychiatry," IEEE Spectrum, vol. 54, no. 7, pp. 45-50, 2017.

[34] M. D. Griffiths, "Internet gambling, player protection, and social responsibility," in Routledge international handbook of Internet gambling, R. J. Williams, R. T. Wood, and J. Parke, Eds. London and New York: Routledge, 2012, ch. 13, pp. 227-249.

[35] D. Miers, "Social responsibility and harm minimization in commercial gambling in great britain," Gaming Law Review and Economics, vol. 20, no. 2, pp. 164-176, 2016. 
[36] Gambling Commission, "Gambling participation in 2016: behavior, awareness and attitudes - Annual report," http://www.gamblingcommission.gov.uk/PDF/survey-data/ Gambling-participation-in-2016-behaviour-awareness-and-attitudes. pdf, 2017, online; accessed 11 Nov. 2017.

[37] E. Korkea-Aho and P. Leino, "Who owns the information held by eu agencies? weed killers, commercially sensitive information and transparent and participatory governance," Common Market Law Review, vol. 54, no. 4, pp. 1059-1091, 2017.

[38] T. C. Lin, "Executive trade secrets," Notre Dame L. Rev., vol. 87, p. 911,2011

[39] N. Hing, L. Cherney, A. Blaszczynski, S. M. Gainsbury, and D. I. Lubman, "Do advertising and promotions for online gambling increase gambling consumption? An exploratory study," International Gambling Studies, vol. 14, no. 3, pp. 394-409, 2014.

[40] J. Metcalf, E. F. Keller, and D. Boyd, "Perspectives on big data, ethics and society," The Council for Big Data, Ethics and Society, 2016.

[41] T. Y. Tang and P. Winoto, "I should not recommend it to you even if you will like it: the ethics of recommender systems," New Review of Hypermedia and Multimedia, vol. 22, no. 1-2, pp. 111-138, 2016.

[42] D. Paraschakis, "Towards an ethical recommendation framework," in 11th International Conference on Research Challenges in Information Science (RCIS). IEEE, 2017, pp. 211-220.

[43] R. Cassidy, A. Pisac, and C. Loussouarn, Qualitative research in gambling: Exploring the production and consumption of risk. Routledge, 2013.

[44] European Parliament and Council, "Regulation (EU) 2016/679 of 27 April 2016 on the protection of natural persons with regard to the processing of personal data and on the free movement of such data, and repealing Directive 95/46/EC (General Data Protection Regulation)," Official Journal of the European Union, p. 88, 2016.

[45] UK Public General Acts, "Gambling Act 2005," http://www.legislation. gov.uk/ukpga/2005/19/pdfs/ukpga_20050019_en.pdf, 2005, online; accessed 11 Nov. 2017
[46] M. D. Griffiths, R. T. Wood, and J. Parke, "Social responsibility tools in online gambling: A survey of attitudes and behavior among internet gamblers," Cyberpsychology \& Behavior, vol. 12, no. 4, pp. 413-421, 2009.

[47] Responsible Gambling Strategy Board, "The national responsible gambling strategy 2016-17 to 2018-19," https://about.gambleaware.org/ media/1230/rgsb_strategy_2016-2019.pdf, 2016, online; accessed 11 Nov. 2017.

[48] M. Soleymani, S. Asghari-Esfeden, M. Pantic, and Y. Fu, "Continuous emotion detection using EEG signals and facial expressions," in IEEE International Conference on Multimedia and Expo (ICME), 2014, pp. $1-6$.

[49] S. Harrison, "Fairer and safer gambling (keynote speech)," in Raising Standards Conference. Gambling Commission, Nov 2017, pp. 17. [Online]. Available: http://www.gamblingcommission.gov.uk/PDF/ speeches/Raising-standards-keynote-speech-Sarah-Harrison-2017.pdf

[50] E. A. Locke and G. P. Latham, "New directions in goal-setting theory," Current Directions in Psychological Science, vol. 15, no. 5, pp. 265-268, 2006.

[51] G. H. Seijts and G. P. Latham, "Learning versus performance goals: When should each be used?" The Academy of Management Executive, vol. 19 , no. 1 , pp. 124-131, 2005.

[52] M. E. Seligman, Helplessness: On depression, development, and death New York: WH Freeman/Times Books/Henry Holt \& Co, 1975.

[53] M. E. Larimer, D. L. Irvine, J. R. Kilmer, and G. A. Marlatt, "College drinking and the greek system: Examining the role of perceived norms for high-risk behavior," Journal of College Student Development, vol. 38, no. 6 , p. 587,1997

[54] G. Eysenbach, "The law of attrition," Journal of Medical Internet Research, vol. 7, no. 1, p. e11, 2005.

[55] L. Yardley, S. Williams, K. Bradbury, G. Garip, S. Renouf, L. Ware, H. Dorling, E. Smith, and P. Little, "Integrating user perspectives into the development of a web-based weight management intervention," Clinical Obesity, vol. 2, no. 5-6, pp. 132-141, 2012. 